\title{
Meningiomas espinales
}

\author{
M. Gelabert-González; A. García-Allut y R. Martínez-Rumbo
}

Servicio de Neurocirugía. Hospital Clínico Universitario. Departamento de Cirugía. Universidad de Santiago de Compostela.

Resumen

Objetivos. Analizar la incidencia, presentación clínica, hallazgos radiológicos, localización, tratamiento y resultados operatorios de los meningiomas espinales.

Pacientes y método. Estudio retrospectivo sobre 57 meningiomas espinales en 55 pacientes operados entre Enero de 1980 y Junio de 2004. Analizamos la edad, sexo, síntomas de debut y duración de los mismos, características radiológicas, técnica quirúrgica, estudios histológicos y resultados.

Resultados. La serie consta de 55 pacientes (46 mujeres y 9 varones), con edades comprendidas entre 12 y 84 años (media 65.2 \pm 9.2 ). El síntoma de debut más frecuentes fue el déficit motor en 34 casos (61.8\%) y la duración media de la sintomatología antes del diagnóstico de $\mathbf{6 . 2}$ meses. La distribución a lo largo del raquis mostró 51 casos en la región dorsal, 5 cervicales y 1 lumbar. El tipo histológico más frecuente fue el meningotelial $(\mathrm{N}=\mathbf{3 1}$, $54.3 \%)$.

Conclusiones. Los meningiomas espinales constituyen en nuestra experiencia el $46 \%$ de todos los tumores intradurales extramedulares y el $7.5 \%$ de todos los meningiomas. Afectan sobre todo a mujeres y en la mayoría de los casos se ubican en la región dorsal. Su localización habitual es intradural extramedular y se desarrollan lentamente extendiéndose lateralmente por el espacio subaracnoideo. La resonancia magnética es la técnica diagnóstica de elección y la resección quirúrgica completa conduce a una recuperación total en la mayoría de los casos.

PALABRAS CLAVE: Cirugía espinal. Laminectomía. Meningioma. Resonancia magnética. Tumor espinal.

\section{Summary}

Objective. To present the incidence, clinical presentation, radiological features, localization, surgical techni-

Recibido: 12-04-05. Aceptado: 19-05-05 ques, and long term results of surgically treated spinal meningiomas.

Patients and methods. We review retrospectively 57 spinal meningiomas in 55 patients operated between January 1980 and june 2004. Age, sex, duration and type of symptoms, radiological and surgical results, histological features and long term outcome are analyzed.

Results. Fifty five patients, consisting $\mathbf{4 6}$ females and 9 males, with an age range from 12 to 84 years (mean 65.2 \pm 9.2 ) were identified. The first symptom was motor deficit $(\mathrm{N}=34,61.8 \%)$ and the mean duration symptoms were: 6.2 months. Distribution along the spinal axis was 51 thoracic, 5 cervical and 1 lumbar. The most common type was meningothelial $(\mathrm{N}=\mathbf{3 1}, \mathbf{5 4 . 3 \%})$.

Conclusions. Meningiomas account in our experience, the $46 \%$ of spinal cord tumours and account the $7.5 \%$ of all meningiomas. They are more frequent in females most frequently occur in the thoracic region and have low recurrence rate. Typically, they are located in the intradural extramedullary space, grow slowly, and spread laterally in the subarachnoid space. Finally we concluded that magnetic resonance imaging is the best imaging technique for diagnosis and total tumour resection improved the surgical results of spinal meningiomas.

KEYWORDS: Laminectomy. Magnetic resonance imaging. Meningioma. Spinal surgery. Spinal tumour.

\section{Introducción}

Los meningiomas espinales son los tumores intradurales y extramedulares más frecuentes, representando en la literatura entre el $20 \%$ y el $46 \%$ del total de los tumores en esta localización ${ }^{10,18,35,39}$. La relación entre meningiomas espinales e intracraneales oscila en las diferentes series entre $1: 2.5$ y $1: 16^{20,25,30,42}$. Típicamente se sitúan en la región dorsal y su localización es intradural extramedular, creciendo lentamente y con tendencia a extenderse lateralmente por el espacio subaracnoideo ${ }^{23}$.

La forma habitual de presentación es con el desarro- 
Tabla 1

Grados de Nurick, pre y postoperatorios

\begin{tabular}{|c|c|c|c|c|c|}
\hline \multirow[t]{2}{*}{ GRADO } & \multirow[t]{2}{*}{ DESCRIPCIÓN } & \multicolumn{2}{|c|}{ PREOPERATORIO } & \multicolumn{2}{|c|}{ POSTOPERATORIO (6 meses) } \\
\hline & & $\mathrm{N}^{\circ}$ Casos & $\%$ & $\mathrm{~N}^{\circ}$ Casos & $\%$ \\
\hline 1 & Movilidad normal & 15 & 27.2 & 29 & 54.1 \\
\hline 2 & $\begin{array}{l}\text { Ligera dificultad para caminar. } \\
\text { Vida doméstica y laboral normal }\end{array}$ & 15 & 27.2 & 13 & 23.9 \\
\hline 3 & $\begin{array}{l}\text { Dificultad moderada para caminar. } \\
\text { Dificulta vida doméstica y laboral. }\end{array}$ & 14 & 25.4 & 6 & 11 \\
\hline 4 & Imposible caminar sin ayuda & 5 & 9 & 3 & 5.5 \\
\hline 5 & $\begin{array}{l}\text { Incapacidad completa para caminar. } \\
\text { Encamado }\end{array}$ & 6 & 10.9 & 3 & 5.5 \\
\hline
\end{tabular}

Tabla 2

Síntomas y signos preoperatorios

\begin{tabular}{|l|l|c|}
\hline & $\mathrm{N}^{\mathrm{o}}$ casos & $\%$ \\
\hline Paraparesia/paraplejia & 34 & $\mathbf{6 1 . 8}$ \\
\hline Dolor radicular & 17 & $\mathbf{3 0 . 9}$ \\
\hline Alteración de esfínteres & 13 & $\mathbf{2 3 . 6}$ \\
\hline Parestesias & 9 & $\mathbf{1 6 . 3}$ \\
\hline Alteración de reflejos & 49 & $\mathbf{8 9}$ \\
\hline Nivel sensitivo & 18 & $\mathbf{3 2 . 7}$ \\
\hline $\begin{array}{l}\text { Sin sintomatología neurológica } \\
\text { (incidental) }\end{array}$ & 1 & $\mathbf{1 . 8}$ \\
\hline
\end{tabular}

Tabla 3

Localización axial del tumor y síntoma de presentación

\begin{tabular}{|l|c|c|c|c|}
\hline & \multicolumn{4}{|c|}{ Síntomas de presentación } \\
\hline Posición & Motor & Sensitivo & Dolor & Total \\
\hline Anterior & 6 & 1 & 1 & 8 \\
\hline Antero-lateral & 11 & 0 & 2 & 13 \\
\hline Lateral & 8 & 1 & 1 & 10 \\
\hline Posterior & 6 & 0 & 3 & 9 \\
\hline Postero-lateral & 3 & 1 & 10 & 14 \\
\hline$N^{\circ}$ pacientes & 34 & 3 & 17 & $54 *$ \\
\hline
\end{tabular}

*una paciente no presentaba sintomatología neurológica (incidental)

llo de signos neurológicos secundarios a la compresión medular o con episodios de dolor radicular ${ }^{20}$. La resonancia magnética permite el diagnóstico exacto en cuanto a su localización, extensión y relaciones con la médula espinal. $\mathrm{Su}$ pronóstico es en general bueno, con una rápida recuperación de los déficits neurológicos tras la cirugía, lo que llevó a Cushing y Eisenhardt en 1938 a indicar que la exéresis de un meningioma espinal: "es uno de los proceso más gratificantes de todos los procedimientos quirúrgi$\cos ^{117}$.

Presentamos nuestra experiencia en el diagnóstico y tratamiento de los meningiomas espinales, analizando una serie consecutiva de 55 pacientes.

\section{Pacientes y métodos}

Entre enero de 1980 y junio de 2004, hemos intervenido quirúrgicamente 412 pacientes con meningiomas de diferentes tipos histológicos y localizaciones, de los cuales 55 (46 mujeres y 9 varones) presentaban 57 meningiomas espinales confirmados patológicamente. Revisamos los datos clínicos básicos, síntomas y tiempo de evolución, técnicas de diagnóstico utilizadas, tratamiento quirúrgico practicado, complicaciones derivadas y resultados. Así mismo, hemos recogido los datos histopatológicos de los tumores extirpados. La funcionalidad motora de los pacientes fue analizada pre y postoperatoriamente, empleando la escala funcional de Nurick $^{32}$ (Tabla 1).

\section{Resultados}

Edad y sexo: las edades oscilaron entre 12 y 84 años (media 65.2 \pm 9.2 ). El 81.8\% de los pacientes (44 casos) se encontraban entre la quinta y octava décadas de la vida. Existía un claro predominio de las mujeres ( $\mathrm{N}=46,83.6 \%)$ sobre los varones.

Antecedentes: tres pacientes habían sido intervenidas 2 , 4 y 20 años antes de una neoplasia de mama y otra lo fue 6 




Figura 1. RM secuencia T1. (Izquierda): corte sagital que muestra un meningioma anterior en D3-D4. (Centro): con gadolinio se observa realce homogéneo. (Derecha) el corte axial con contraste muestra la localización anterior del tumor y la compresión medular.

meses después del diagnóstico de meningioma espinal.

Otra paciente había sido intervenida por nosotros 5 años antes de un meningioma parietal. Un paciente varón de 16 años, diagnosticado de neurofibromatosis tipo II, fue operado 5 años antes de un meningioma frontal, desarrollando posteriormente múltiples meningiomas intracraneales, 3 schwannomas y 3 meningiomas espinales.

Sintomatología: el déficit motor fue el primer síntoma en la mayoría de los pacientes, $(\mathrm{N}=34,61.8 \%)$ seguido por el dolor radicular $(\mathrm{N}=17,30.9 \%)$. Alteraciones esfinterianas aparecían en 13 pacientes (23.6\%) (Tabla 2). La duración de la sintomatología osciló entre 10 días y 84 meses (media: 6.2 meses) (Tabla 3). Una paciente de la serie debutó con una hemorragia subaracnoidea con angiografía negativa; ante el desarrollo posterior de una paraparesia, se practicó un estudio mielográfico que condujo al diagnóstico ${ }^{16}$. En otra paciente el diagnóstico fue incidental, al realizarse un estudio de resonancia magnética cerebral por problemas otológicos.

Se realizó estudio del líquido cefalorraquídeo en 18 pacientes, observándose en 11 casos (61\%) elevación de la cifra de proteínas, con el estudio citológico normal.

Estudios radiológicos: en 10 casos (18.1\%) (anteriores a 1988) el diagnóstico fue realizado mediante mielografía, en la que se apreciaba un bloqueo completo en 7 casos $(70 \%)$, y parcial en los otros $3(30 \%)$. Los restantes 45 pacientes fueron diagnosticados con resonancia magnética, donde se apreciaba en T1 una tumoración que se comportaba en todos los casos como isoseñal con la médula y realce homogéneo, tras la administración de contraste (Figura 1).

Localización del tumor: en la región cervical se localizaron 5 tumores (8.7\%), 51 eran dorsales (89.4\%), y 1 lumbar (1.9\%). La localización exclusivamente intradural se observó en 56 meningiomas y únicamente 1 era extradural (1.7\%). En la distribución axial, 8 meningiomas eran anteriores (14\%), 13 anterolaterales $(22.9 \%), 11$ posteriores $(15.8 \%), 15$ posterolaterales $(26.3 \%)$ y laterales 10 $(17.5 \%)$. El paciente de 16 años con neurofibromatosis tipo II presentaba 3 meningiomas intradurales torácicos posteriores.

Tratamiento quirúrgico: excepto 4 pacientes, con meningioma cervical, que fueron operados en posición sentada, todos los casos fueron intervenidos en decúbito prono practicando una laminectomía estándar, que en algunas ocasiones fue necesario ampliar hasta las apófisis articulares. Se realizó exéresis completa mediante técnica microquirúrgica clásica en 54 casos. En 6 pacientes fue necesario resecar la duramadre de implantación y en los otros 49 se electrocoaguló la base sobre la que se asentaba el tumor. En 13 pacientes fue preciso emplear duramadre liofilizada para reparar el defecto dural y en todos los casos se selló la sutura dural con adhesivos de cianocrilato o fibrina.

Histología: se realizó estudio anatomopatológico en todos los tumores resecados (57 meningiomas). Las formas histológicas más frecuentes fueron la meningotelial 
$(\mathrm{N}=31,54.3 \%)$, y la psamomatosa $(\mathrm{N}=16,28 \%)$. Los otros 10 tumores fueron informados únicamente como meningiomas, sin especificar la variedad.

Complicaciones: una paciente falleció a los 6 días de la cirugía por una embolia pulmonar. Cuatro casos presentaron una fístula de líquido cefalorraquídeo precisando una segunda intervención para el cierre de la misma. Dos casos presentaron infección local de la herida quirúrgica que fue tratada médicamente. Así mismo, 31 pacientes presentaron infección urinaria.

Resultados y seguimiento: excepto la paciente fallecida, al alta todos habían mejorado clínicamente en relación a su estado inicial (Tabla 1). El tiempo de seguimiento de los pacientes osciló entre 10 meses y 15 años (media 7.3 años). Excepto una paciente de 63 años con un meningioma extradural cervical localizado en la cara anterior, en la que se realizó una extirpación incompleta y que presentó un recrecimiento al año de la cirugía, no hemos tenido hasta la fecha ningún caso de recidiva. Durante el seguimiento, once pacientes fallecieron de otras causas no relacionadas con el meningioma espinal.

\section{Discusión}

Los meningiomas espinales son tumoraciones histológicamente benignas, que representan entre el 25 y $46 \%$ de todos los tumores extramedulares e intradurales y con una incidencia anual calculada de 8 casos por millón de habitantes ( 5 mujeres y 3 varones) $)^{1,17,18,23,28,31,39}$. En nuestra experiencia constituyen el $12.1 \%$ de todos los meningiomas operados entre 1980 y 2004 (412 casos); con una relación 1:7.8 entre meningiomas espinales-intracraneales.

Aunque existen algunas referencias sobre meningiomas espinales en niños ${ }^{9,17}$, se trata de tumoraciones típicas de adultos y ancianos, siendo en nuestra casuística la edad media al diagnóstico de 65.2 años, ligeramente superior a otras series publicadas, como 53 años en la de Levy et $\mathrm{al}^{25}$, 56 años para Solero et al ${ }^{43}$ o 57 para Klekamp y Samii ${ }^{24}$.

En nuestra casuística la relación mujer-varón fue de 5: 1, y el $81.8 \%$ de los pacientes tenían entre 40 y 80 años. En la serie de Levy et al ${ }^{25}$ esta relación fue de 4:1; ascendiendo hasta 8:1 en la revisión de Solero et al ${ }^{43}$ y de 9:1 en la de Poisson et $\mathrm{al}^{36}$. Numerosos autores indican que el meningioma tiene una mayor frecuencia en las mujeres en edad fértil, debido a una posible dependencia estrogénica de los mismos ${ }^{42}$. Sin embargo, esta relación es controvertida, y los estudios histológicos han demostrado la presencia de receptores hormonales, no sólo para estrógenos sino también para esteroides, progesterona, andrógenos y prolactina $^{13,36,41}$. (En nuestra serie 3 mujeres habían sido intervenidas previamente de un cáncer de mama, un tumor con una relación estrogénica bien conocida).

En la literatura existen múltiples referencias entre embarazo y progresión de meningiomas intracraneales, recalcando el papel hormonal en el desarrollo de estos tumores, pero esta relación con los meningiomas espinales es poco frecuente, probablemente, porque se trata de mujeres de mayor edad ${ }^{4,27}$.

Los estudios genéticos muestran una pérdida completa o parcial del cromosoma 22 en más del $50 \%$ de los casos de meningiomas espinales ${ }^{18}$. También se ha publicado la relación de los genes 1p, 9p, 10q, y 17q con meningiomas espinales $^{2,20}$. Ketter et al ${ }^{22}$ indican que la existencia de anomalías cromosómicas no presuponen un comportamiento más agresivo ni una mayor tendencia a las recidivas, como ocurre con los meningiomas intracraneales, donde la presencia de múltiples anomalías cromosómicas supone un mayor riesgo de recidiva.

Los meningiomas espinales son tumores de origen mesodérmico que se originan en las células aracnoideas próximas a las raíces espinales, creciendo lentamente en sentido lateral sobre el espacio subaracnoideo hasta que se hacen sintomáticos ${ }^{17,25}$. Al contrario que los meningiomas craneales, los espinales no penetran en la piamadre, debido probablemente a la existencia de una "capa leptomeníngea intermedia" entre la piamadre y la aracnoides; otra explicación para este fenómeno es que al tratarse de tumores que dan sintomatología con pequeño tamaño y que por lo tanto la extirpación quirúrgica es habitualmente más precoz que en los craneales, no transcurre el suficiente tiempo para que el tumor pueda penetrar en la pia ${ }^{40}$. Aunque en la mayoría de las series el dolor radicular o vertebral es el síntoma más frecuente ${ }^{24,25,39}$ en nuestros pacientes predominaron los datos de afectación neurológica (motor o sensitiva), apareciendo en segundo lugar los síntomas dolorosos.

Menos habitual es la presentación con datos clínicos de hipertensión intracraneal (cefalea, alteraciones visuales), producidos por el bloqueo en la reabsorción del líquido cefalorraquídeo, debido al incremento de proteínas licuorales, obstrucción venosa o hemorragias tumorales ${ }^{25}$.

En nuestra experiencia, la localización más frecuente es en la región dorsal (90\%), siendo poco habituales los lumbares. Cohen-Gadol et $\mathrm{al}^{5}$ encuentran en su serie que los pacientes menores de 50 años tienen una mayor incidencia de meningiomas cervicales (39\%), sobre todo en la región cervical superior. En nuestra serie, solamente 5 pacientes tenían menos de 50 años y en todos ellos el meningioma era de localización dorsal. Levy et $\mathrm{a}^{25}$ opinan que la topografía del tumor guarda relación con el sexo, con una mayor incidencia dorsal en las mujeres ( $83 \%$ ); además encuentran que los meningiomas cervicales están en el $84 \%$ de los casos en situación ventral.

Los meningiomas espinales son, en general, tumores únicos aunque en la literatura existen numerosas referencias a casos múltiples ${ }^{15,38} \mathrm{o}$ asociados a otros tumores medulares (schwannomas, neurofibromas) ${ }^{11}$, como nuestro 
paciente de 16 años afecto de NF tipo II y que, además de 3 meningiomas espinales, presentaba múltiples meningiomas intracraneales y 3 schwannomas raquídeos.

Aunque se han descrito casos de localización extradural, intra-extradurales e incluso intramedulares ${ }^{6}$, la mayoría de los meningiomas tienen una localización puramente intradural ${ }^{15}$. En nuestra serie no existía ningún tipo de "meningioma en placas", una variedad de escasa incidencias en la que el tumor crece en forma anular alrededor de la duramadre, tiene tendencia a infiltrar la aracnoides y, por lo tanto, una mayor incidencia de recidivas ${ }^{3}$.

En nuestra serie, observamos en el estudio analítico del líquido cefalorraquídeo la presencia de disociación albúmino-citológica, encontrándose que la proteinorraquia estaba elevada en el $61 \%$ de los pacientes en los que fue estudiada, cifras ligeramente inferiores a otras series de la literatura donde llegan a suponer hasta el $90 \%$ de los $\operatorname{casos}^{25}$.

La mielografía, empleada por nosotros para el diagnóstico de los 10 casos más antiguos de la serie, tiene hoy en día un papel histórico, ya que la resonancia magnética es la técnica diagnóstica de elección, al indicarnos en un solo estudio la localización exacta del tumor, la extensión y situación axial del mismo y la presencia de otras lesiones asociadas como tumores o cavidades siringomiélicas ${ }^{34}$. En la mayoría de los casos los meningiomas espinales son isointensos con la médula espinal en secuencias T1 y T2, con un intenso realce homogéneo tras la administración de gadolinio ${ }^{33}$; en algunas ocasiones es posible visualizar un realce periférico similar a la "cola" descrita en los meningiomas intracraneales ${ }^{37}$. La RM en cortes axiales con gadolinio es esencial para conocer las relaciones del tumor, su situación con respeto a la médula espinal y facilitar la planificación quirúrgica.

La resección completa del tumor es posible en la mayoría de las ocasiones, siendo para Levy et al., en el $82 \%$ de los $\operatorname{casos}^{25}$, en el $97 \%$ para King et $\mathrm{al}^{23}$ y en el $98.7 \%$ para Roux et $\mathrm{al}^{39}$. La mayor dificultad técnica depende de la localización axial del tumor, sobre todo en aquellos situados en la cara anterior ${ }^{15}$, aunque en estos casos, la sección de los ligamentos dentados suele permitir un desplazamiento seguro de la medula para acceder al tumor. Gambardella et $\mathrm{a}^{14}$ publican una serie de 10 pacientes con meningiomas de la región dorsal situados ventralmente y operados por un abordaje postero-lateral combinado con un abordaje transpedicular-transarticular; obtienen buenos resultados en el $80 \%$ de los casos. Algunos autores recomiendan el empleo preoperatorio de ultrasonidos para una mejor localización del tumor y apuntando entre otras ventajas una menor extensión de la laminectomía y una reducción en la apertura dural ${ }^{26}$.

Se discute la actitud a seguir con la base de implantación; en los situados posteriormente es factible la resec- ción de la base y su reemplazo por una plastia dural; sin embargo, en los situados ventralmente esta opción es más difícil por lo que la mayoría de los autores recurren a su electrocoagulación. Solero et $\mathrm{a}^{43}$ no encuentran diferencias significativas en cuanto a recidivas con el empleo de una u otra opción.

En general, la mayoría de los pacientes tienen una buena recuperación funcional después de la cirugía, y los índices de deterioro neurológico son bajos, incluso en aquéllos con gran afectación motora preoperatoria, siempre que se siga un buen programa de rehabilitación ${ }^{20} \mathrm{e}$ independientemente de su edad ${ }^{29}$. La morbilidad y mortalidad quirúrgica es muy baja y en la mayoría de las series ésta se encuentra por debajo del $3 \%{ }^{18,25}$, y casi siempre esta relacionada con patología previa del paciente o complicaciones operatorias, como la fístula de LCR que puede conducir a infecciones meníngeas ${ }^{24,39}$. Histológicamente las variedades encontradas a nivel espinal son similares a los intracraneales; los subtipos meningotelial y psamomatoso son los más frecuentes, siendo excepcionales las variedades malignas o anaplásicas, aunque existen algunos casos descritos de transformación maligna ${ }^{12}$.

La evolución de los meningiomas espinales es mucho mejor que los meningiomas intracraneales, y las recidivas en la mayoría de las series está por debajo del $10 \%^{23,25,40,42}$. El lento crecimiento de estos meningiomas, asociado a su presentación en pacientes de edad avanzada contribuye a este bajo índice de recidivas ${ }^{23}$. Levy et $\mathrm{al}^{25} \mathrm{y} \mathrm{Rath}^{38}$ indican que los meningiomas extradurales tienen una mayor tendencia a la recidiva por su carácter más invasivo y una mayor velocidad de progresión. En nuestra serie, únicamente existe un caso de recidiva en el grupo de 40 pacientes con más de 5 años de seguimiento en una mujer con un meningioma cervical de localización ventral y extradural, previamente operada de un cáncer de mama. Cohen-Gadol et $\mathrm{al}^{5}$, encuentran que los índices de recidivas y reoperaciones es mayor en los pacientes menores de 50 años, debido a una mayor presencia de meningiomas cervicales, gran incidencia de extradurales y tendencia a crecer en placas, factores que dificultan la extirpación quirúrgica. Deen et $a{ }^{8}$ publican también una mayor incidencia de recidivas (20\%) en pacientes menores de 21 años. La presencia de calcificaciones también se considera como factor de peor pronóstico y Levy et a ${ }^{25}$ comunican una mala evolución en 3 de los 4 casos que presentaban calcificaciones.

Algunos autores indican la necesidad de radioterapia en las recidivas, aunque, si el estado del paciente y la morfología de la recidiva lo permiten es preferible, en nuestra opinión, la reintervención, ya que se trata de un tumor histológicamente benigno y sólo debería recurrirse a la irradiación si existe una grave contraindicación quirúrgica ${ }^{20,21}$. Gezen et a ${ }^{19}$ publican en su serie 2 casos de recidiva tratados con radioterapia y sin evidencia de pro- 
gresión posterior. Roux et al ${ }^{39}$ también trataron 2 casos de recidiva con radiocirugía consiguiendo la estabilización del tumor, sin progresión en 5 años.

\section{Conclusiones}

Los meningiomas espinales son tumores benignos que afectan sobre todo a mujeres en la edad media de la vida y con una mayor incidencia en la región dorsal. La RM es el método diagnóstico de elección, muy útil para la planificación quirúrgica y para el seguimiento postoperatorio.

Histológicamente, la mayoría de los meningiomas espinales son meningoteliales o psamomatosos, aunque las diferencias histológicas no influyen en el pronóstico.

La cirugía es el método óptimo para el tratamiento, y se acompaña de una excelente recuperación funcional, incluso en pacientes con gran afectación neurológica previa.

\section{Bibliografía}

1. Albanese, V., Platania, N.: Spinal intradural extramedullary tumors J Neurosurg Sci 2000; 46: 18-24.

2. Arslantas, A., Artan, S., Oner, U., et al.: Detection of chromosomal imbalances in spinal meningiomas by comparative genomic hybridization. Neurol Med Chir 2003; 43: 1219.

3. Caroli, E., Acqui, M., Roperto, R., Ferrante, L., D'Andrea, G.: Spinal en plaque meningiomas: A contemporary experience. Neurosurgery 2004; 55: 1275-1279.

4. Cioffi, F., Buric, J., Carnesecchi, S., Romoli, S., Conti, P.: Spinal meningiomas in pregnancy: report of two cases and review of the literature. Eur Gynaecol Oncol 1996; 17: 384388.

5. Cohen-Gadol, A.A., Zikel, O.M., Koch, C.A., Scheithauer, B.W., Krauss, W.E.: Spinal meningiomas in patients younger than 50 years of age: a 21-year experience. J Neurosurg 2003; 98 (suppl 3): 258-263.

6. Covert, S., Gandhi, D., Goyal, M., Woulfe, J., Belanger, E., Miller, W., Modha, A.: Magnetic resonance imaging of intramedullary meningioma of the spinal cord: case report and review of the literature. Can Assoc Radiol J 2003; 54: 177180.

7. Cushing, H., Eisenhardt, L.: Meningiomas: Their classification, regional behaviour, life history and surgical end results. Charles C. Thomas, Springfield. 1938.

8. Deen, H.G. Jr., Scheithauer, B.W., Ebersold, M.J.: Clinical and pathological study of meningiomas of the first two decades of life. J Neurosurg 1982; 56: 317-322.

9. Di Rocco, C., Ianelli, A., Colosimo, C.: Spinal epidural meningiomas in childhood: a case report. J Neurosurg Sci 1994; 38: 251-254.

10. Domínguez, J., Rivas, J.J., Lobato, R.D., Gómez, P.A., Alday, R., Cabrera, A., Rivero, B., Rodríguez, C., Boto, G.R.:
Meningiomas espinales. Revisión de 40 casos. Neurocirugía 1998; 9: 123-128.

11. Dorizzi, A., Crivelli, G., Marra, A., Scamoni, C., Dario, A., Bonfanti, N., Brianza, M.L.: Associated cervical schwannoma and dorsal meningioma. Case report and review of the literature. J Neurosurg Sci 1992; 36: 173-176.

12. Endo, K., Tanaka, S., Komagata, M., et al: Rhabdoid transformation of recurrent meningioma in the cervical cord: a case report. J Orthop Sci 2004; 9: 323-326.

13. Gabos, S., Berkel, J.: Meta-analysis of progestin and estrogen receptors in human meningiomas. Neuroepidemiology 1992; 11: 255-260.

14. Gambardella, G., Toscano, S., Staropoli, C., Longo, M., D'Amico, D., Marafioti, T., Tomasello, F.: Epidural spinal meningioma. Role of magnetic resonance in differential diagnosis. Acta Neurochir (Wien) 1990; 107: 70-73.

15. Gambardella, G., Gervasio, O., Zaccone, C.: Approaches and surgical results in the treatment of ventral thoracic meningiomas. Review of our experience with a posterolateral combined transpedicular-transarticular approach. Acta Neurochir (Wien) 2003; 145: 385-392.

16. Gelabert, M., Prieto, A., Bollar, A., García, A.: Hemorragia subaracnoidea secundaria a tumor espinal. Rev Neurol 1992; 104: 151-154.

17. Gelabert, M., Cutrín, J.M., González, J.: Meningioma espinal en la infancia. Rev Esp Pediatría 1999; 55: 281-283.

18. Gelabert, M., García, A., Fernández, J.M.: Meningiomas espinales. Neurología 2000; 15: 58-62.

19. Gezen, F., Kahraman, S., Canakci, Z., Bedük. A.: Review of 36 cases of spinal cord meningioma. Spine 2000; 25: 727-31.

20. Gottfried, O.N., Gluf, W., Quiñones-Hinojosa, A., Kan, P., Schmidt, M.H.: Spinal meningiomas: surgical management and outcome. Neurosurg Focus 2003; 14: 1-7.

21. Gottfried, O.N., Schmidt, M.H.: Management of spinal meningiomas. Contemporary Neurosurg 2003; 25: 1-4.

22. Ketter, R., Henn, W., Niedermayer, I., Steilen-Gimbel, H., Konig, J., Zang, K.D.: Predictive value of progressionassociated chromosomal aberrations for the prognosis of meningiomas: a retrospective study of 198 cases. J Neurosurg 2001; 95: 601-607.

23. King, A.T., Sharr, M.M., Gullan, R.W., Bartlett, J.R.: Spinal meningiomas: a 20-year review. Br J Neurosurg 1998; 12: $521-526$.

24. Klekamp, J., Samii, M.: Surgical results of spinal meningiomas. Acta Neurochir (Wien) 1996; 65: 77-81.

25. Levy, W.J., Bay, J., Dohn, D.: Spinal cord meningioma. J Neurosurg 1982; 57: 804-812.

26. Maiuri, F., Iaconetta, G, Gallicchio, B., Stella, L.: Intraoperative sonography for spinal tumors. Correlations with MR findings and surgery. J Neurosurg Sci 2000; 44: 115122.

27. Mealey, J. Jr., Carter, J.E.: Spinal cord tumor during 
pregnancy. Obstet Gynecol 1969; 32: 204-209.

28. Mirimanoff, R.O., Dosretz, D.E., Lingood, R.M., Ojemann, R.G., Martuza, R.L.: Meningioma: Analysis of recurrente and progresión following neurosurgical resection. J Neurosurg 1985; 62: 18-24.

29. Morandi, X., Haegelen, C., Riffaud, L., Amlashi, S., Adn, M., Brassier, G.: Results in the operative treatment of elderly patients with spinal meningiomas. Spine 2004; 29 : 2191-2194.

30. Namer, I.J., Pamir, M.N., Benli, K., Saglam, S., Erbengi, A.: Spinal meningiomas. Neurochirurgie 1987; 30: 11-15.

31. Nelson, J.S., Parisi, J.E., Schochet, S.S.: Principles and practice of neuropathology. In: Parisi JE, Mena H (eds). Non-Glial tumors. Mosby-Year Book Inc. St Louis, 1993; pp. 203-213.

32. Nurick, S.: The pathogenesis of the spinal cord disorder with cervical spondylosis. Brain 1972; 95: 87-100.

33. Pardo, J., Lema, M.: Meningiomas espinales. En: Lema M, Gelabert M. (eds). Meningiomas. Santiago de Compostela: Servicio de publicaciones de la Universidad de Santiago de Compostela, 2002; pp. 263-275.

34. Pérez, M.I., Burriel-Rosello, P.A., Marin-Cardenas, M.A., Pascual-Piazuelo, Alfaro-Tomas, J.: Coexistencia de siringomielia y meningioma intradural extramedular. Rev Neurol 2004; 39: 142-145.

35. Prevedello, D.M.S., Koerbel, A., Esteves, C., Truite, L., Grande, C.V., da Silva, L.F., Araújo, J.C.: Prognostic factors in the treatment of the intradural extramedullary tumors: a study of 44 cases. Arq Neuropsiquiatr 2003; 61: 241-247.

36. Poisson, M., Magdelenat, H., Pertuiset, B.F.: Les récepteurs de steroids de méningiomes intracraniens. Neurochirurgie 1986; 32 (suppl 1) 63-69.
37. Quekel, L.G., Versteege, C.W.: The "dural Tail Sign" in MRI of spinal meningiomas. J Comput Assist Tomogr 1995; 19: 890-892.

38. Rath, S., Mathai, K.V., Candí, J.: Multiple meningiomas of the spinal canal. Case report. J Neurosurg 1967; 26: 639640 .

39. Roux, F.X., Nataf, F., Pinaudeau, M., Borne, G., Devaux, B., Meder. J.F.: Intraspinal meningiomas: Review of 54 cases with discussion of poor prognosis factors and modern therapeutic management. Surg Neurol 1996; 46: 458-464.

40. Salpietro, F.M., Alafaci, C., Lucerna, S., Iacopino, D.J., Tomasello, F.: Do spinal meningiomas penetrate the pial layer? Correlation between magnetic resonance imaging and microsurgical findings and intracranial tumor interfaces. Neurosurgery 1997; 41: 254-258.

41. Schick, U., Marquardt, G., Lorenz, R.: Recurrence of benign spinal neoplasms. Neurosurg Rev 2001; 24: 20-25.

42. Schlehofer, B., Blettner, M., Wahrendorf, J.: Association between brain tumors and menopausal status. J Nall Cancer Inst 1992; 84: 1346-1349.

43. Solero, C., Fornari, M., Giombini, S., Lasio, G., Oliveri, G., Cimino, C., Pluchino, F.: Spinal meningiomas: Review of 174 operated cases. Neurosurgery 1989; 25: 153 160.

Gelabert-González, M.; García-Allut, A.; MartínezRumbo, M.: Meningiomas espinales. Neurocirugía 2006; 17: $125-131$.

Correspondencia postal: M. Gelabert González. Servicio de Neurocirugía. Hospital Clínico Universitario. La Choupana, s/n. 15706 Santiago de Compostela 